Laser Chem. 1988, Vol. 8, pp. 315-334

(C) 1988 Harwood Academic Publishers GmbH

Photocopying permitted by license only

Reprints available directly from the Publisher

Printed in the United Kingdom

\title{
Experimental and Theoretical Analysis of Non-linear Vibrational Relaxation of Polyatomic Molecules Strongly Excited by Resonant Laser Radiation
}

\section{CARLOMUSTO and A. CARTELLI}

Facolta' di Ingegneria, Universita' di Cassino, Via Zamosch 03043, Cassino, Italy

\author{
S. SOLIMENO, R. VELOTTA and R. BRUZZESE $†$ \\ Dipartimento FNSMFA Pad. 20 Mostra d'Oltremare, 80125 Napoli, Italy
}

We present a very simple theoretical model aimed at the analysis of non-linear relaxation processes in molecular gases in the presence of partial molecular dissociation induced by vibrational-vibrational exchange between highly excited molecules. The model has a phenomenological character, since it analyzes the behavior of a system of anharmonic diatomic molecules, which is a very rough approximation of a polyatomic molecule such as $\mathrm{SF}_{6}$. Nonetheless, it provides an interesting key for the interpretation of a number of peculiar features characterizing our experimental observation, with which a comparison is made. In particular, the model takes realistic account of the influence of dissociation processes on the relaxation time.

KEY WORDS: Multiphoton dissociation.

\section{INTRODUCTION}

Collisional relaxation from vibrationally excited states of molecules plays an important role in many physical phenomena, including isoto- 
pically selective dissociation, lasing in gases, chemical kinetics and transport properties (see, for example Refs. 1-3, and papers cited therein).

Thus, it is of importance to study vibrational relaxation processes and to have knowledge of the relaxation times of molecular systems, in particular of those far from the equilibrium.

One of the interesting aspects of the research on the vibrational relaxation is the investigation of the decay of strongly excited states. The relaxation time, $\tau_{\mathrm{VT}}$, of a simple harmonic oscillator depends on the kinetic temperature, $T$, according to the Landau-Teller $\operatorname{law}^{2} \tau_{\mathrm{VT}} \propto$ $\exp \left(T^{-1 / 3}\right)$. For strong excitations the temperature $T$ can change notably during the relaxation process, producing a corresponding variation of $\tau_{\mathrm{VT}}$. This effect was predicted and verified experimentally. ${ }^{2,11}$

When the excitation energy is increased, moreover, dissociation of the molecules can occur, ${ }^{1}$ thus making the dependence of $\tau_{\mathrm{VT}}$ on the energy more intricate than that predicted by the simple Landau-Teller law. Thus, in the case of very strong excitation, non-linear relaxation depends on the rate of excitation. Consequently, in highly excited systems one can expect a change in the effective relaxation time with the laser flux and a deviation of the vibrational temperature law from the simple exponential decay.

The dependence of the rate of vibrational relaxation on the excitation intensity can have considerable influence on many photophysical and photochemical processes. A typical example is the process of multiphoton dissociation of molecules in the presence of collision. ${ }^{4}$ Moreover, it is possible to use non-linear processes in vibrational relaxation to increase the selectivity of optical excitation.

For the above reasons it is of great interest to investigate in detail various non-linear relaxation mechanisms.

In a number of previous papers ${ }^{5.6}$ we have reported studies of the non-linear behavior of the VT relaxation time of pure $\mathrm{SF}_{6}$ and of $\mathrm{SF}_{6}$ and a buffer gas in a regime of strong laser excitation (up to $1.5 \mathrm{~J} / \mathrm{cm}^{2}$ ). In particular, we have observed a strong time dependence of $\tau_{\mathrm{VT}}$ during the relaxation process, and its noticeable decrease in correspondence of laser energy fluxes $\left(\geq 1 \mathrm{~J} / \mathrm{cm}^{2}\right)$ at which multiphoton dissociation (MPD) processes have been observed. ${ }^{7}$ Thus, our results have confirmed in a very clear way the strong dependence of $\tau_{\mathrm{VT}}$ on the energy flux. 
A particularly interesting feature of our result is that the non-linear relaxation process that we have observed occurs with a timedependent relaxation time, which shows a clear stepwise behavior: it is characterized by a fast initial rate followed by a slower second rate. This stepwise behavior of $\tau_{\mathrm{VT}}(t)$ and other characteristic features of our results described in Ref. 6 confirmed the relaxation law in the presence of dissociation processes predicted previously. ${ }^{8.5}$

In Ref. 6 we also presented the outline of a very simple, phenomenological model aimed at the analysis of non-linear relaxation in gases in the presence of partial molecular dissociation induced by vibrational-vibrational (VV) exchange between vibrationally highly excited molecules. We present here the complete, and greatly improved version of the above model. In particular, in its final version our model is now capable of numerically integrating the rate equations for the various molecular vibrational levels taken into account, by using non-approximate expressions for the transition probabilities of VV exchange. Moreover, we take full account of the gas temperature variations during the relaxation process, thus obtaining a direct estimate for the time dependence of the physical quantity which is directly linked to what we actually measure during the experiments, as will be described in Section II where we briefly discuss the experimental method, apparatus and results.

Although our simple theoretical model, which is fully discussed in the third section, represents a very rough approximation of a polyatomic molecule such as $\mathrm{SF}_{6}$, and is still phenomenological in its character, it offers, nonetheless, an interesting key for the interpretation of our experimental observations, and explains their most important features. In particular, it has the merit of taking realistically into account the influence of the dissociation processes on the VT relaxation time, a feature which we were the first to observe and report in the literature (see Ref. 6 and references quoted therein).

The comparison between experimental and theoretical results and our conclusions are given in Section IV.

\section{EXPERIMENTAL TECHNIQUE AND RESULTS}

In the experiments we have used a very sensitive technique originally developed for studying the absorption of small amounts of energy by 
gases and liquids. ${ }^{9}$ In this technique, named phase fluctuation optical heterodine (PFLOH) spectroscopy, the small phase change of a single frequency laser, which results when it passes through a transparent medium whose density and, consequently, refractive index have been changed by energy absorption, is detected.

The experimental apparatus has been fully described in previous works $^{5,6}$ and, thus, we shall only report here its main features.

A gas cell, containing the sample under investigation, is placed in one arm of a Mach-Zehnder interferometer, illuminated by a linearly polarized, single mode $\mathrm{HeNe}$ laser. The excitation laser is a linetunable TEA $\mathrm{CO}_{2}$ laser delivering up to $5 \mathrm{~J}$ per pulse $(\sim 100 \mathrm{~ns}$ FWHM) on each line. Both $\mathrm{CO}_{2}$ (directed and focused by a set of gold-coated mirrors) and $\mathrm{HeNe}$ overlap in the sample zone, with the $\mathrm{CO}_{2}$ laser inducing the refractive index change and $\mathrm{HeNe}$ laser beam probing it. A photodiode detects the heterodyned signal, which is then fed into a storage oscilloscope for the final analysis.

The interferometer is mounted on a honeycomb table provided with a pneumatic isolation system for guaranteeing vibration isolation and improving its stability.

A more thorough discussion of the PFLOH technique and of a number of conditions which have to be satisfied in order to apply it to VT relaxation studies can be found in Ref. 6. We limit ourselves to report that in this method the measured quantity is the phase change difference $\Delta \Phi(t)$ between the laser beams in the two arms of the interferometer:

$$
\Delta \Phi(t)=\frac{2 \cdot \pi \cdot l}{\lambda} \cdot\left(n_{0}-1\right) \cdot \frac{\Delta T}{T_{0}}
$$

where $l$ is the cell length, $\lambda$ the HeNe laser wavelength, $\Delta T$ the induced temperature variation, $T_{0}$ and $n_{0}$ the initial gas temperature and refractive index at $T=T_{0}$ (room temperature), respectively.

$\tau_{\mathrm{VT}}$ can, then, be directly inferred from the oscillograms of $\Delta \Phi(t)$ (see Ref. 6).

By using the experimental setup and technique just described, we have studied the temporal evolution of the VT relaxation during the relaxation process, its frequency and flux dependence in the regime of strong laser excitation, and the influence of a buffer gas on the process. ${ }^{6}$ In the following we shall briefly report the main features of 
this experimental analysis in order to carry out a clear comparison with the predictions of the numerical model presented in the next section.

In the $\mathrm{SF}_{6}$ molecule (a spherical top) the absorption of IR laser photons around $10.6 \mu \mathrm{m}$ takes place through the IR active, $v_{3}=948$ $\mathrm{cm}^{-1} F_{1 u}$ vibrational mode. Experimental results suggest that in a regime of low excitation the relaxation process is characterized by a single, constant relaxation time ${ }^{10}$ which is given by $\bar{\tau}_{\mathrm{VT}}=100 / p$ ( $\mu$ s/torr), where $p$ is the $\mathrm{SF}_{6}$ pressure. ${ }^{2}$

At higher excitation energies $\left(\gtrsim 50 \mathrm{~mJ} / \mathrm{cm}^{2}\right)$, the anharmonicity of individual modes and gas heating can cause non-linearities in the thermalization of excited molecular gases, as firstly observed in the pioneering work by Akhmanov et al. ${ }^{11}$ The anharmonicity of molecular vibrations has the effect that even in the case of diatomic molecules the thermalization of a gas as a result of the VT relaxation is nonexponential: the characteristic relaxation time strongly depends on the energy absorbed by the gas. The resulting heating of the gas accelerates the VT relaxation process in accordance with the Landau-Teller law. ${ }^{4}$ In what follows we shall indicate this non-linear, time-dependent relaxation time with $\tau_{\mathrm{VT}}{ }^{A}(t)$.

Since $\tau_{\mathrm{VT}}{ }^{A}(t)$ shows a strong time dependence, ${ }^{6}$ in order to characterize the relaxation process at high laser fluxes we introduce an effective relaxation time $\tau_{\text {eff }}$, which is defined as the time in which the initial deviation of the gas temperature from the equilibrium value changes by a factor of $1 / e$.

Firstly, we have carefully analyzed the oscillograms of $\Delta \Phi(t)$ under multiline and single-line excitation conditions for pure $\mathrm{SF}_{6}$ and $\mathrm{SF}_{6}$ buffer gas mixtures, on a very broad range of laser fluxes (from 10 $\mathrm{mJ} / \mathrm{cm}^{2}$ to $1.5 \mathrm{~J} / \mathrm{cm}^{2}$ ). In the case of low flux (up to $\sim 50 \mathrm{~mJ} / \mathrm{cm}^{2}$ ) the relaxation process is still linear, i.e., it is characterized by a fairly constant relaxation time. At higher flux the features of our experimental results change completely. Figure 1 shows two typical oscillograms of $\Delta \Phi(t)$ for 2 torr of pure $\mathrm{SF}_{6}$ excited by the $\mathrm{CO}_{2}$ laser tuned on the 10P16 line $\left(947.7 \mathrm{~cm}^{-1}\right)$. In case (a), with a flux of about $0.25 \mathrm{~J} / \mathrm{cm}^{2}$, the relaxation process is strongly non-linear, and the relative $\tau_{\text {eff }}$ is about $50 \mu$ s. In case (b), with a higher flux of $\sim 1 \mathrm{~J} / \mathrm{cm}^{2}$, the process is characterized by a shorter $\tau_{\text {eff }}(\sim 40 \mu \mathrm{s})$, and a new feature is observed. In fact, the non-linear relaxation process occurs with a time dependent relaxation time which shows a stepwise behavior: the oscilloscope signal is clearly characterized by a initial faster rate, which has an 


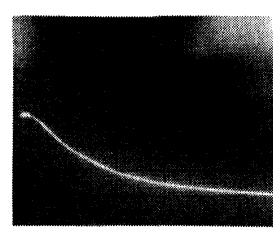

a

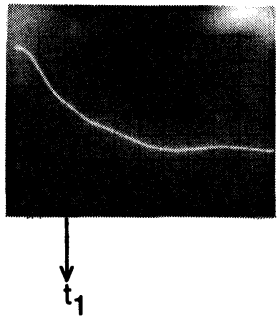

b

Figure 1 Oscillograms of $\Delta \Phi(t)$ for 2 torr of pure $\mathrm{SF}_{6}$ excited by the 10P16 line of the $\mathrm{CO}_{2}$ laser for two different fluxes: $0.25 \mathrm{~J} / \mathrm{cm}^{2}$ (case a) and $1.0 \mathrm{~J} / \mathrm{cm}^{2}$ (case b). The base line passes through the maximum on the left $(20 \mu \mathrm{sec} / \mathrm{div})$.

important effect in determining $\tau_{\text {eff }}$, and a second slower rate. The change in the slope takes place, as indicated in Figure 1b, after a time $t_{1}$ $\simeq 20 \mu \mathrm{s}$. This effect will be discussed in Section IV. We would like to mention here, however, that the signal of Figure 2 have been observed many times and in different experimental conditions. In particular, the stepwise behavior of case (b) has always, and only, been observed in



Figure 2 Flux dependence of $\tau_{\text {eff }}$ for three different laser lines (2 torr of pure $\mathrm{SF}_{6}$ ). 
correspondence of very high fluences and correspondingly very short relaxation times.

It is worth noticing that in the oscillograms of Figure 1 the $\mathrm{CO}_{2}$ laser pulse is right at the beginning (left part) of the photograph, i.e. at $t=0$, while the time required for the signal to recover back to baseline is of several ms.

In Figure 2 the effective relaxation time is reported as a function of the laser flux, $F$, for different laser lines. The points of Figure 2 are only a fraction of a large number of measurements carried out at different pressures and laser lines, but all showing the same trend: the relaxation time is a decreasing function of the laser flux. It is worth noting at this point that for energy fluxes higher than $\sim 1.5 \mathrm{~J} / \mathrm{cm}^{2}$ the interferometric technique cannot give anymore correct result with the path length used. In fact, because of the high excitation energy $\Delta T=T(t)-T_{0}$ assumes very large values and, consequently, the condition $\Delta \Phi(t) \ll 1$, which is one of the conditions to be satisfied in the PFLOH technique, is not met. The corresponding oscillograms show the expected oscillating behavior.

As a result Figure 2 shows the strong dependence on the laser flux of the relaxation time: $\tau_{\text {eff }}$ is a decreasing function of the laser flux.

\section{THEORETICAL ANALYSIS}

As already reported in the introduction, in the simple model of Ref. 6 we obtained an analytical expression for the effective relaxation time, $\tau_{\text {eff }}$, in $\mathrm{SF}_{6}$ in the presence of partial molecular dissociation induced by VV exchange between vibrationally highly excited molecules. In fact the final expression of $\tau_{\text {eff }}$ was arrived at without solving explicitly the system of rate equations for the various molecular vibrational levels, and by using approximate expressions for the transition probabilities of VV and VT exchange.

In what follows we describe a model that starting from the same simple and restrictive assumptions of the analysis of Ref. 6, obtains numerical values for $\tau_{\text {eff }}$, as a function of the time and of the vibrational energy initially stored into the molecules, by direct integration of the appropriate vibrational rate equations for the system. Moreover, the approximation in calculating the transition probabilities for $\mathrm{VV}$ and VT energy exchange is also removed. 


\section{i. Main assumptions}

We analyze a system of one-dimensional truncated anharmonic oscillators vibrationally excited by intense laser radiation that is almost resonant with the first vibrational level of the IR active mode of the molecules (the mode $v_{3}$ for $\mathrm{SF}_{6}$ ).

Since in a highly excited $\mathrm{SF}_{6}$ molecule independent modes are strongly coupled, the energy accumulated in the $v_{3}$ mode will be rapidly distributed amongst the other modes without the need for collisions. ${ }^{12}$ This fast intramolecular VV exchange takes place on a time scale, of the order of the picoseconds, which is much shorter than the time characterizing the collision-induced intermolecular VV thermalization at 1 torr $(\sim 1 \mu \mathrm{s}) .{ }^{4,12}$ The subsequent VT process couples the vibrational modes to the translations in about $100 \mu \mathrm{s}$ at the same pressure. Thus, since the $v_{6}$ vibrational mode has the lowest quantum energy $\left(363 \mathrm{~cm}^{-1}\right)$ and, consequently, the highest transition probability, it is reasonable to assume that the VT relaxation process proceeds mainly through the $v_{6}$ mode.

The above considerations justify our drastic approximation of assimilating the relaxation of $\mathrm{SF}_{6}$ molecules to the relaxation of anharmonic oscillators with a $0 \rightarrow 1$ transition frequency of $363 \mathrm{~cm}^{-1}$, i.e., coincident with that of the $v_{6}$ mode. The anharmonicity is accounted for by the use of a Morse potential for the oscillators. ${ }^{13}$

In order to take collision-induced dissociation processes into account, the anharmonic oscillator energy is truncated to the $n^{\text {th }}$ level; i.e., we assume that $X_{n}=0$, where $X_{n}$ is the population of the $n^{\text {th }}$ vibrational level. Thus, a fraction of the molecules will spend its energy for dissociating. It is assumed in the following that only a small fraction of the molecules undergoes dissociation. Furthermore, given the laser fluxes we are experimentally interested in $\left(\leq 1.5 \mathrm{~J} / \mathrm{cm}^{2}\right)$, laser-induced unimolecular dissociation processes are disregarded in our model.

The dissociation of a vibrationally excited anharmonic oscillator $(A B)^{*}$ induced by a collision with another vibrationally excited oscillator $\left(A^{\prime} B^{\prime}\right)^{*}$ can be described by the following equation:

$$
(A B)^{*}+\left(A^{\prime} B^{\prime}\right)^{*} \rightarrow\left(A^{\prime} B^{\prime}\right)^{+}+A+B+\Delta H
$$

where $\Delta H$ is the enthalpy variation of the system, and $\left(A^{\prime} B^{\prime}\right)^{+}$denotes the ground state or a state characterized by a lower (vibrational) energy content. For the dissociation process described by Eq. (2) $\Delta H$ 
is generally positive. The absolute value of $\Delta H$ depends, mainly, on the vibrational state in which $\left(A^{\prime} B^{\prime}\right)$ is left after the collision. At any rate, the dissociation process ${ }^{2}$ beyond opening up a new channel to the relaxation process, can cause a faster heating of the molecular gas because of the instantaneous release of the energy $\Delta H$ in a collision. This will be taken into account in our model.

Finally, we shall only consider one-quantum transitions, thus assuming vanishingly small the probability of collisional multiquantum transitions.

\section{ii. Rate equations}

In analyzing vibrational excitation in molecular collisions the region of interest is that of small energies of translational motion $(\leqslant 1 \mathrm{eV})$. In this case, the condition of the adiabatic approximation is usually satisfied (with respect to electronic and translational motion). This condition can be written as ${ }^{2}$

$$
\tau_{\text {coll }} \gg \tau_{\mathrm{el}} \simeq \hbar / \Delta E_{\mathrm{el}}
$$

Moreover, most molecules satisfy the adiabaticity condition with respect to electronic and vibrational motion: ${ }^{2}$

$$
\tau_{\mathrm{vib}} \simeq \hbar / \Delta E_{\mathrm{vib}} \gg \tau_{\mathrm{el}}
$$

In Eqs. (3) and (4), $\tau_{\text {coll }} \simeq a / v$ is the characteristic collision time, where $a$ is the effective radius of action of the intermolecular forces, and $v$ is the molecular mean velocity; $\tau_{\mathrm{el}}$ and $\tau_{\mathrm{vib}}$ are the characteristic times for electronic and vibrational motion; $\Delta E_{\mathrm{el}}$ and $\Delta E_{\mathrm{vib}}$ are the energies of the electronic and vibrational transitions; and, finally, $\hbar$ is the Plank constant divided by $2 \pi$.

In the adiabatic approximation of Eqs. (3) and (4) we need not to account explicitly for electronic motion (it is always taken into account implicitly in the intermolecular interaction potential). We need to treat only the translational, rotational, and vibrational degrees of freedom. Here molecular collisions are represented by collisions between rotating anharmonic oscillators, which usually satisfy well the following conditions:

$$
\tau_{\text {coll }} \gg \tau_{\text {vib }}, \quad \tau_{\text {coll }}<\tau_{\text {rot }} \sim \hbar / \Delta E_{\text {rot }}
$$


where $\tau_{\text {rot }}$ is the characteristic time for rotational motion, and $\Delta E_{\text {rot }}$ the corresponding transition energy. Simultaneous fulfillment of these conditions implies that the probability of exciting rotational levels is considerably greater than the probability of vibrational excitation. Hence, in studying vibrational relaxation we can assume that equilibrium between translational and rotational degrees of freedom has already been established. In what follows we shall, thus, neglect any other process but vibrational relaxation.

In order to know the temperature variation as a function of time we have to solve the rate equations for $N+1$ levels ( 0 being the ground state, and $N$ the dissociation level) of an anharmonic oscillator. We shall, thus, be able to calculate the temporal evolution of the population distribution in the different vibrational levels and, as a consequence, the quantity of vibrational energy transformed in translational temperature (gas heating).

We have considered in the rate equations system the following processes: (a) intermolecular VV exchange (characterized by the time $\tau_{\mathrm{VV}}$ ); (b) VT exchange (with characteristic time $\tau_{\mathrm{VT}}$ ); and (c) dissociation processes.

Since the VV and VT relaxation processes are temporally well separated, i.e., $\tau_{\mathrm{VV}} \ll \tau_{\mathrm{VT}}$ we can assume that as a consequence of the fast VV exchange (process a) a "quasi-equilibrium" takes place. We shall discuss the problem of this initial population distribution in the following paragraph of this section.

As far as process (c) is concerned, we notice that since we are considering only one-quantum transitions, only molecules in the vibrational level $\mathrm{N}$ will undergo dissociation processes.

By defining with $N_{0}$ and $Z$, the total number of molecules and the rate of collisions between molecules respectively, we can write the rate equations for the population of the ground level (0), the $n^{\text {th }}$ vibrational level $(n=1,2, \ldots, N-1)$, and the dissociation level $N$ as follows,

$$
\begin{aligned}
\frac{d}{d t} X_{0}= & Z\left(P_{1,0} X_{1}-P_{0,1} X_{0}\right)+\frac{Z}{N_{0}} \\
& \cdot\left[\sum_{m=0}^{N-1}\left(Q_{1,0}^{m, m+1} X_{m} X_{1}-Q_{0,1}^{m+1, m} X_{m+1} X_{0}\right)\right] ;
\end{aligned}
$$




$$
\begin{aligned}
\frac{d}{d t} X_{n}= & Z\left(P_{n+1, n} X_{n+1}-P_{n, n+1} X_{n}-P_{n, n-1} X_{n}+P_{n-1, n} X_{n-1}\right) \\
& +\frac{Z}{N_{0}}\left[\sum _ { m = 0 } ^ { N - 1 } \left(Q_{n+1, n}^{m, m+1} X_{m} X_{n+1}-Q_{n, n+1}^{m+1, m} X_{m+1} X_{n}\right.\right. \\
& \left.\left.-Q_{n, n-1}^{m, m+1} X_{m} X_{n}+Q_{n-1, n}^{m+1, m} X_{m+1} X_{n-1}\right)\right] \\
\frac{d}{d t} X_{N}= & Z\left(P_{N-1, N} X_{N-1}-P_{N, N-1} X_{N}\right) \\
& +\frac{Z}{N_{0}}\left[\sum _ { m = 0 } ^ { N - 1 } \left(Q_{N-1, N}^{m+1, m} X_{m+1} X_{N-1}\right.\right. \\
& \left.\left.-Q_{N, N-1}^{m, m+1} X_{m} X_{N}\right)\right]-P_{d} \cdot Z \cdot X_{N}
\end{aligned}
$$

$P_{n, n+1}$ is the probability of transition from the level $n$ to $n+1 ; Q_{n, k}^{i, j}$ (with $j=i+1$ and $k=n-1$ ) is the probability per collision of the transition of the interacting molecules from the levels $i$ and $n$ to the levels $j$ and $k$ respectively; $P_{d}$, finally, is the dissociation probability. All these three quantities will be discussed in the next paragraphs.

From the principle of detailed balancing we have:

$$
\begin{aligned}
P_{n, n+1} & =P_{n+1, n} \cdot \exp \left[-\left(E_{n+1}-E_{n}\right) / T\right] \\
Q_{n, n+1}^{m+1, m} & =Q_{n+1, n}^{m, m+1} \cdot \exp \left[-\left(E_{n+1}-E_{n}+E_{m}-E_{m+1}\right) / T\right]
\end{aligned}
$$

where the energy $E_{n}$ of the $n^{\text {th }}$ vibrational level is expressed in Kelvin $\left({ }^{\circ} \mathrm{K}\right)$. Since we are considering Morse oscillators we have:

$$
E_{n}=(n+1 / 2) \cdot E_{1}-(n+1 / 2)^{2} \cdot \Delta E
$$

with $E_{1}=h v_{6}\left(\right.$ for $\left.\mathrm{SF}_{6}\right), \Delta E=\chi E_{1}$, and $\chi=E_{1} /(4 \cdot D)$, where $D$ is amplitude characterizing the Morse potential. 
By using Eq. (8) we can recast Eqs. (6a-c) as:

$$
\begin{aligned}
\frac{d}{d t} X_{0}= & Z \cdot P_{1,0}\left\{X_{1}-X_{0} \cdot \exp \left[-\left(E_{1}-2 \Delta E\right) / T\right]\right\} \\
& +\frac{Z}{N_{0}}\left\{\sum _ { m = 0 } ^ { N - 1 } Q _ { 1 , 0 } ^ { m , m + 1 } \left[X_{m} X_{1}-X_{m+1} X_{0}\right.\right. \\
& \cdot \exp (-2 m \Delta E / T)]\} \\
\frac{d}{d t} X_{n}= & Z \cdot\left\{P_{n+1, n}\left[X_{n+1}-X_{n} \cdot \exp \left\{-\left[E_{1}-Z(n+1) \Delta E\right] / T\right\}\right]\right. \\
& -P_{n, n-1}\left(X_{n}-X_{n-1} \exp \left[-\left(E_{1}-2 n \Delta E\right) / T\right]\right) \\
& +\frac{Z}{N} \cdot\left\{\sum _ { m = 0 } ^ { N - 1 } \left[Q_{n+1, n}^{m, m+1} X_{m} X_{n+1}-X_{m+1} X_{n}\right.\right. \\
& \cdot \exp \{-[2(m-n) \Delta E] / T\})-Q_{n, n-1}^{m, m+1}\left(X_{m} X_{n}-X_{m+1} X_{n-1}\right. \\
& \cdot \exp \{-[Z(m-n+1) \Delta E] / T\})]\} \\
\frac{d}{d t} X_{N}= & -Z \cdot P_{N, N-1}\left(X_{N}-X_{N-1} \exp \left[-\left(E_{0}-2 N \Delta E\right) / T\right]\right) \\
& -\frac{Z}{N_{0}} \cdot\left[\sum _ { m = 0 } ^ { N - 1 } Q _ { N , N - 1 } ^ { m , m + 1 } \left(X_{m} X_{N}-X_{m+1} X_{N-1}\right.\right. \\
& \cdot \exp [2(m-N+1) \Delta E / T])]-P_{d} Z X_{N}
\end{aligned}
$$

This is the set of equations to be solved numerically in order to obtain the time evolution of the gas temperature variation, as we shall show in the last paragraph of this section.

\section{iii. Initial population distribution and transition probabilities}

Let us consider a system of $N_{0}$ molecules at the temperature $T$, excited by intense laser radiation almost resonant with the first vibrational 
level of the IR active mode of the molecules (e.g., $v_{3}$ for $\mathrm{SF}_{6}$ ). Because of the vibrational excitation of the molecules the system will be in an unstable state. In this condition the fast VV exchange process establishes a "quasi steady-state" vibrational distribution ${ }^{2}$ in a very short time $(\sim 1 \mu$ s at 1 torr $)$, and before the actual relaxation takes place $\left(\tau_{\mathrm{VV}}\right.$ $\left.\ll \tau_{\mathrm{VT}}\right)$.

This distribution was derived by Treanor et al. ${ }^{14}$ and has the following expression:

$$
N_{n}=N_{0} \cdot \exp \left[-n E_{1} / T_{v 1}+\left(n E_{1}-E_{n}\right) / T\right]
$$

where $N_{n}$ is the number of molecules with energy $E_{n}\left(n^{\text {th }}\right.$ vibrational level), and $T_{\text {vib }}$ the vibrational temperature of the first vibrational level (energy $E_{1}$ ).

As we can see from Eq. (10) in a system of anharmonic oscillators the "quasi steady-state" distribution established by the fast VV exchange is no longer a Boltzmann distribution, as in the case of harmonic oscillators (where $E_{n}=n \cdot E_{1}$ ).

An important condition which has to be met in order Eq. (10) to hold true is that the total number of vibrational quanta of the system is conserved during the $\mathrm{VV}$ processes. This is true as long as $\tau_{\mathrm{VV}} \ll \tau_{\mathrm{VT}}$, a condition surely met for the lower vibrational levels.

As for the use of a Treanor distribution, for the vibrational energy stored into the molecules we would like to stress here that we are referring to a situation where collisions play a fundamental role: in our model we suppose that a Treanor-type distribution is established after the fast $(<1 \mu \mathrm{s})$, intermolecular (i.e., collisionally induced) VV exchange between the anharmonic oscillators has taken place. Contrary to this, in the analysis of collisionless multiphoton energy deposition and dissociation processes, a Boltzmann-type energy distribution has been suggested. ${ }^{15,16,17}$ This refers to the distribution of the vibrational energy in $\mathrm{SF}_{6}$ molecules arising from IR irradiation in experimental conditions where, because of the use of molecular beams ${ }^{15,16}$ or of very dilute $\left(<10^{-3}\right.$ torr) gas pressures, ${ }^{17}$ the collisional relaxation rates measured for $\mathrm{SF}_{6}$ are unimportant, as clearly stated in Ref. 17. This is a condition, obviously, very different from the one we are analyzing.

A point which is worth commenting upon at this stage is that the Treanor distribution of Eq. (10), that we have used as initial vib- 
rational distribution for the population of the $N+1$ levels, is a function not only of $T$, but also of $T_{\text {vib }}$, i.e., of the vibrational temperature of the first level. The value of $T_{\mathrm{vib}}$ is linked to the amount of energy absorbed by the system. The higher $T_{\text {vib }}$, the higher the energy stored in the molecules as vibrational energy, as can be easily seen by calculating the average energy per molecule as a function of $T_{\mathrm{vib}} \cdot{ }^{18} \mathrm{On}$ the other hand, at least in the laser energy range we have investigated, the energy absorbed per molecule is an increasing function of the laser flux. As a consequence, by solving the system of Eqs. $(9 \mathrm{a}-\mathrm{c})$ with initial Treanor distributions characterized by different values of $T_{\text {vib }}$ we shall be able to calculate $\tau_{\text {eff }}$ as a function of the vibrational energy initially stored into the molecules, namely, as a function of the laser flux. It will, thus, be possible to make a comparison with the experimental data reported in Figure 2, at least on a qualitative base.

We close this paragraph with a short discussion of the vibrational transition probabilities appearing in the Eqs. $(9 \mathrm{a}-\mathrm{c})$.

For the probabilities of a transition from level $n$ to $n+1\left(P_{n+1, n}\right)$, and of a transition of two colliding molecules from levels $m$ and $n+1$ to the levels $m+1$ and $n$ respectively $\left(Q_{n+1 ; n}^{m, m+1}\right)$, we have used the following expression (see Ref. 2 and references quoted therein):

$$
\begin{aligned}
P_{n+1, n}= & V_{n+1, n}^{2} \cdot Z_{0}^{-1} \frac{0.163 \cdot \mu T}{\alpha^{2}} f\left(\gamma_{n}\right) \exp (\epsilon / T) \exp \left(\Delta E_{n} / 2 T\right) \\
Q_{n+1, n}^{m, m+1}= & V_{m, m+1}^{2} V_{n+1, n}^{2} \frac{0.163 \cdot \mu \mathrm{T}}{\alpha^{2}} \\
& \cdot Z_{0}^{-1} \cdot f\left(\gamma_{m n}\right) \exp (\epsilon / T) \exp \left(\Delta E_{m n} / 2 T\right)
\end{aligned}
$$

where $\mu$ is the reduced mass of the colliding particles (in atomic units), $\alpha$ the constant in the exponential intermolecular interaction potential $\sim e^{-} e^{\alpha \pi}$ (in $\AA^{-1}$ ), $\Delta E_{n}$ and $\Delta E_{m n}$ are the changes in the vibrational energy in the transitions (in ${ }^{\circ} \mathrm{K}$ ), $Z_{0}$ is an orientation factor which for polyatomic molecules takes a value between 1.5 and 10 (see Ref. 2), $f\left(\gamma_{n}\right)$ and $f\left(\gamma_{m n}\right)$ are adiabatic factors whose full expressions can be found in Ref. 2 , and $V_{m n}^{2}$ is the mean square value of the matrix element for the transition $n \rightarrow m$, for a full discussion of which the 
reader is again referred to Ref. 2 . The factor $\exp (\epsilon / T)$, where $\epsilon\left(\right.$ in $\left.{ }^{\circ} \mathrm{K}\right)$ is the depth of the well in the intermolecular potential, takes approximate account of the attraction between the colliding particles, while the factor $\exp \left(-\Delta E_{m n} / 2 T\right)$ accounts for the change in relative velocity of the particles upon collision.

With reference to these vibrational probabilities a point which is worth commenting upon is their dependence on the translational gas temperature $T$. We have taken full account of this temperature dependence in the numerical solution of Eqs. $(9 a-c)$ by calculating at each integration step the new translational temperature $T$ and using this new value for updating the transition probabilities to be used in the next integration step.

\section{iv. Numerical results}

By numerically solving the set of Eqs. $(9 \mathrm{a}-\mathrm{c})$ we have calculated, as a function of the time, the gas temperature in the interaction volume during the relaxation process. The value of the temperature variation at the $n^{\text {th }}$ integration step $\Delta T(n)$ is given by:

$$
\Delta T(n)=T(n)-T_{0}=\frac{\Delta E_{\mathrm{cal}}(n)}{C_{p}}
$$

where $C_{p}$ is the gas thermal capacity at constant pressure and $\Delta E_{\text {cal }}(n)$ the amount of vibrational energy transformed into gas heating in the $n^{\text {th }}$ step:

$$
\begin{aligned}
\Delta E_{\mathrm{cal}}(n)= & E_{\mathrm{vib}}(n-1)-E_{\mathrm{vib}}(n)+\left\{\sum _ { m = 0 } ^ { N } \left[X_{m}(n-1)\right.\right. \\
& \left.\left.-X_{m}(n)\right]\right\} \cdot \Delta H
\end{aligned}
$$

In Eq. (14) $E_{\mathrm{vib}}=\sum_{m=0}^{N} E_{m} X_{m}$ is the total amount of vibrational energy stored into the gas system at each step, and the quantity into square brackets gives the number of molecules that have undergone dissociation during the $n^{\text {th }}$ integration step, thus contributing to the gas heating ( $\Delta H$ different from 0$)$. 
Since, as already discussed in Section II, the relaxation process we are interested in is strongly non-linear, we shall define the effective relaxation time, $\tau_{\text {eff }}$, of our system as the time in which the gas temperature variation reaches the value:

$$
\Delta T\left(\tau_{\text {eff }}\right)=\Delta T(\infty)(1-1 / e)=\left(T_{\infty}-T_{0}\right)(1-1 / e)
$$

where $T_{\infty}$ is the maximum temperature reached by the gas in the excitation region after the VT relaxation process has taken place $(t \gg$ $\left.\tau_{\mathrm{VT}}\right)$.

We have particularly investigated the effect of a partial dissociation of the molecules $\left(P_{d}\right)$, of the amount of energy released in each dissociation process $(\Delta H)$, and of the initial vibrational temperature $T_{\text {vib }}$ which appears in the Treanor distribution (see Eq. (10)).

A first important result of our model is that without introducing a certain degree, even small ( $\sim 5 \div 10 \%)$, of dissociation, corresponding to values of $P_{d}$ ranging between 0.05 and 0.1 , we have been unable to obtain for the time dependence of $\Delta T$, anything that might resemble what we observed in our experiments (see Figure 1b), whatever high the value given to $T_{\mathrm{vib}}$, i.e. to the initial degree of vibrational excitation of the molecules. Moreover, also the value taken on by $\Delta H$ plays an important role. The combined effect of these two key parameters is well illustrated by the results of Figure 3 , where we report $\Delta T$ as a function of time, with a fixed value of $T_{\text {vib }}\left(800^{\circ} \mathrm{K}\right)$, for $P_{d}=0$, i.e. no dissociation, in case (a), and $P_{d}=0.1(10 \%$ dissociation) with $\Delta H=$ $1000^{\circ} \mathrm{K}$ case (b) and $\Delta H=1500^{\circ} \mathrm{K}$ case (c). The difference between the calculated $\tau_{\text {eff }}$ 's (through Eq. (15)) in case (b) and (c) is more than $20 \%$, thus showing the influence of $\Delta H$ on $\tau_{\text {eff }}$.

The plots of Figure 3 have again to be compared to the oscillogram of Figure $1 b$, namely a relaxation process at high laser flux. As seen, only in cases (b) and (c) our model is able to predict a behavior that is qualitatively similar to the stepwise behavior of Figure $1 \mathrm{~b}$.

Our model has also allowed us to make another interesting comparison with experimental results. As already mentioned in the previous paragraph of this section it is possible to choose different values for the vibrational temperature $T_{\text {vib }}$ of the "quasi-steady" initial vibrational population distribution. On the other hand, the energy absorbed per molecule is an increasing function of the laser flux. In particular, in Ref. 19 it has been shown that

$$
T_{\text {vib }} \propto F^{2 / 3}
$$






a)

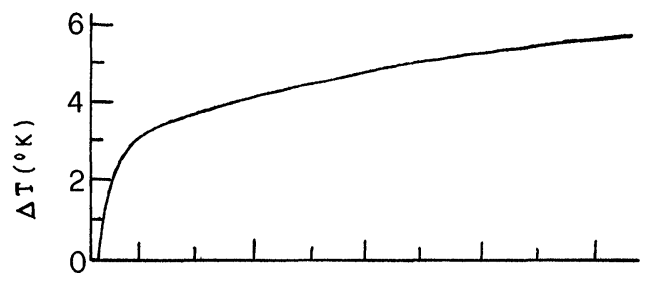

b)

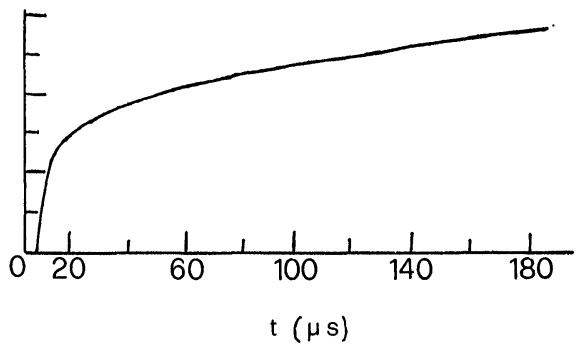

c)

Figure 3 Gas temperature change, $\Delta T$, as a function of time for $P_{d}=0$ case (a), and $P_{d}$ $=0.1$ case ( $\mathrm{b}$ and c), for 2 torr of $\mathrm{SF}_{6} . \Delta H=1000^{\circ} \mathrm{K}$ in case $(\mathrm{b})$, and $\Delta H=1500^{\circ} \mathrm{K}$ in case (c). $\mathrm{T}_{\text {vib }}=800^{\circ} \mathrm{K}$ in all curves.

over a large range of laser fluxes (up to the dissociation threshold). Thus, although the relationship is not linear, we can safely assume that the higher the laser flux the higher $T_{\text {vib. }}$. As a consequence, by solving the system of Eqs. $(9 \mathrm{a}-\mathrm{c})$ with different values of $T_{\text {vib }}$ we have calculated $\tau_{\text {eff }}$ as a function of the vibrational energy initially stored into the molecules. The results are shown in the curve of Figure 4, in the case of $P_{d}=0.01$ and $\Delta H=1000^{\circ} \mathrm{K}$, for 2 torr of pure $\mathrm{SF}_{6}$. This curve can be compared to the plots of Figure 2, where $\tau_{\text {eff }}$ is reported as a function of $F$. As seen, the agreement, at least on a qualitative base, is very good, and $\tau_{\text {eff }}$ shows to be a strongly decreasing function of $T_{\text {vib }}$, a characteristic which is always present in our experimental results. 


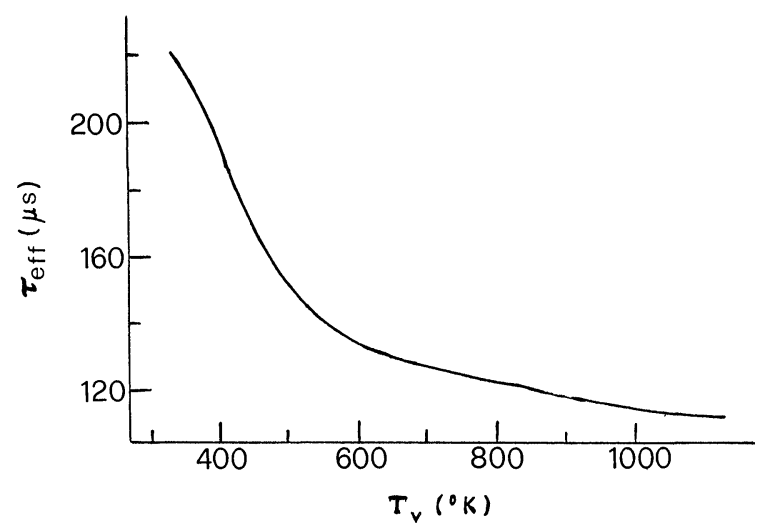

Figure $4 \tau_{\text {eff }}$ as a function of the initial (just after laser irradiation) vibrational temperature $T_{\mathrm{vib}}$. This simulation is relative to $P_{d}=0.1$ and $\Delta H=1000^{\circ} \mathrm{K}$, for 2 torr of pure $\mathrm{SF}_{6}$.

\section{DISCUSSION AND CONCLUSIONS}

Before briefly discussing the numerical results presented in the previous section we think that it is necessary to stress the following. The model we have developed is, obviously, an oversimplified schematization of the process of vibrational relaxation of a polyatomic molecule. We have actually analyzed the behavior of a system of anharmonic diatomic molecules (just a one-dimensional vibrational mode), which is a very rough approximation of a complex molecule such as $\mathrm{SF}_{6}$. Thus, the aim of our analysis is not so much to obtain a complete simulation of the relaxation process, a pretty difficult task, but, rather, to test numerically the effect on the relaxation of a small number of key parameters which are showed to be of importance in our previous measurements. In particular, we have tried to assess the influence of the relaxation of (a) the degree of initial vibrational excitation of molecules; (b) the occurrence of collisionally induced dissociation processes during the relaxation; (c) the amount of heat released by a molecule in a dissociation process, if any.

In particular, as for point (b) we would like to stress that we do not have direct experimental evidence of the fact that dissociation processes do take place (because of our limited experimental facilities). However, the laser flux and intensity of our experiments $\left(\sim 1.5 \mathrm{~J} / \mathrm{cm}^{2}\right.$, 
with $\sim 100 \mathrm{~ns}$ pulse duration) are higher than the threshold power at which induced photofragmentation of $\mathrm{SF}_{6}$ has been observed (see Refs. 17 and 20). Finally, we repeat again that we are hypothesizing a dissociation process which is the result of a collision between vibrationally highly excited molecules, and not the result of a unimolecular process.

Bearing in mind what above specified, we can say that the results of our numerical study provide strong support for the main conclusions of our experimental analysis of non-linear relaxation of $\mathrm{SF}_{6}$ molecules strongly excited by laser radiation, ${ }^{5,6}$ i.e., the fact that $\tau_{\text {eff }}$ is a strongly decreasing function of the laser flux, and the stepwise behavior of $\tau_{\text {eff }}$ in experimental conditions where dissociation processes might be present. In particular, our numerical results have clearly shown that it is impossible to obtain for the calculated value of $\Delta T$ as a function of time (see Figure 3 ) anything which is comparable to our experimental observations (at high laser flux (Figure 1b)), if one does not introduce in the model the possibility of molecular dissociation $\left(P_{d}\right)$ induced by collisions between vibrationally highly excited molecules. In fact, the stepwise behavior of Figure $3 b$ and $3 c$ qualitatively very similar to that observed on the oscilloscope, is clearly not observed in Figure 3a, where $P_{d}=0$, i.e., dissociation processes are forbidden. Also the amount of energy released in each dissociation process is of importance. This is clearly observed in Figure $3 \mathrm{~b}$ and $3 \mathrm{c}$, where a change in $\Delta H(\sim 30 \%)$ causes a correspondent and relevant $(\sim 20 \%)$ change in the inferred value of $\tau_{\text {eff }}$. We like to underline that the possibility of dissociation and the value of $\Delta H$ particularly influence the initial behavior of $\Delta T(t)$, because they allow a faster heating of the system via the instantaneous (through a single collision) release of an amount of energy equal to $\Delta H$.

As for the actual, numerically calculated, values of $\tau_{\text {eff }}$, we have again to stress that we did not aim to predict precise values of relaxation times, but rather to check in what conditions we were able to observe behaviors qualitatively in good agreement with our experimental results. Thus, the values of $\tau_{\text {eff }}$ reported in Figure 4 (ranging between $220 \mu \mathrm{s}$ and $120 \mu \mathrm{s}$ ) are about twice the values measured (see Figure 2) in correspondence of laser fluxes that can produce initial vibrational temperatures comparable to those of Figure 4.

In conclusion, we can safely say that although the model presented here is phenomenological in character and, thus, essentially able to 
allow only a qualitative comparison with experimental results, it has the merit of taking into realistic account the influence of dissociation processes on the VT relaxation process. Moreover, it is able to confirm the strong dependence of the relaxation time of pure $\mathrm{SF}_{6}$ molecules on the laser flux, and to give numerical values for $\tau_{\text {eff }}$ which are not too far from the ones actually measured (within a factor two, which is not too bad if one takes into account its drastic simplifications). The numerical results we have presented, thus, provide a strong support for the analysis, we have recently reported, ${ }^{5,6}$ of vibrational-translational relaxation processes of polyatomic molecules in gas form strongly excited by intense, pulsed, laser radiation, namely, of vibrational relaxation in a regime of strong excitation and in presence of dissociation processes.

\section{References}

1. R. V. Ambartsumyan and V. S. Letokhov, in "Chemical and biochemical applications of lasers," C. B. Moore (ed.), (Academic, N.Y., 1977), Vol. 3, 167.

2. B. F. Gordiets, A. I. Osipov, E. V. Stupochenko and L. A. Shelepin, Sov. Phys. Usp. 15, 759 (1973).

3. S. Ormonde, Rev. Mod. Phys. 47, 193 (1975).

4: V. S. Letokhov, "Non-linear Laser Chemistry," (Springer-Verlag, Berlin, 1983). P. Avouris, M. M. T. Loy and I. Y. Chan, Phys. Lett. 66, 624 (1979).

5. R. Bruzzese, C. d'Ambrosio and S. Solimeno, Infrared Phys. 25, 227 (1985).

6. C. Z. Pan, R. Bruzzese, S. Solimeno and R. Velotta, Jour. Opt. Soc. Am. 134, 452 (1987).

7. P. Kolodner, C. Winterfield and S. Yablonovitch, Opt. Comm. 20, 119 (1977).

8. V. Y. Panchenko, Lett. Nuovo Cimento 39, 380 (1984).

9. C. C. Davis and S. J. Petuchowski, Appl. Phys. 20, 2593 (1981).

10. V. M. Gordienko, A. V. Mikheenko and V. Y. Panchenko, Sov. Techn. Phys. Lett. 5, 186 (1979).

11. S. A. Akhmanov, V. M. Gordienko, V. V. Lazarev, A. V. Mikheenko and V. Y. Panchenko, Sov. Phys. JETP 51, 1087 (1980).

12. A. Fahr and R. D. Bates Jr., Chem. Phys. 105, 449 (1986).

13. E. E. Nikitin, "Theory of elementary atomic and molecular processes in gases," Clarendon Press (Oxford, 1974).

14. C. E. Treanor, J. W. Rich and R. G. Rehm, J. Chem. Phys. 48, 1978 (1968).

15. J. G. Black, P. Kolodner, M. J. Schultz, E. Yablonovitch and N. Bloembergen, Phys. Rev. A19, 704 (1979).

16. P. A. Schultz, Aa. S. Sudb $\varnothing$, E. R. Grant, Y. R. Shen and Y. T. Lee, J. Chem. Phys. 72, 4985 (1980).

17. J. W. Hudgens and J. D. McDonald, J. Chem. Phys. 76, 173 (1982).

18. W. Fuss and J. Hartmann, J. Chem. Phys. 70, 5468 (1979).

19. C. D. Cantrell, "Multiple-photon excitation and dissociation of polyatomic molecules," Springer-Verlag (Berlin, 1985).

20. S. Mucamel and J. Jortner, J. Chem. Phys. 65, 5204 (1976). 